\title{
Flow, heat and mass transfers during solidification under traveling/rotating magnetic field
}

\author{
Wang Xiaodong ${ }^{1} \cdot$ Fautrelle Yves $^{2} \cdot$ Moreau René $^{2} \cdot$ Etay Jacqueline $^{2} \cdot$ \\ Bianchi Ana-Maria ${ }^{3} \cdot$ Baltaretu Florin $^{3} \cdot \mathrm{Na} \mathrm{Xianzhao}^{4}$
}

Received: 9 December 2014/ Accepted: 29 May 2015/Published online: 10 June 2015

(c) The Author(s) 2015. This article is published with open access at Springerlink.com

\begin{abstract}
In this paper, we present the relative problem of heat and mass transfer to adopt a means of imposing an electromagnetic field to improve solutal segregation (macrosegregation) during liquid metal solidification. A well-validated, quasi-two-dimensional solidifying experimental benchmark was introduced, which allowed us to observe the temperature field evolution and to provide the evident clues of phase transformation. We also observed naturally formed solutal segregations in the post-mortem sample. The idea of imposing a modulated magnetic field while optimizing modulation frequency and having a cable to suppress solutal segregation was confirmed by multiscale numerical modeling. Magnetohydrodynamics, flow driven by a modulated traveling magnetic field, was experimentally studied. Furthermore, a more practical cylindrical shape of liquid metal bulk driven by a permanent helical magnetic field has been achieved. The spatial flow behaviors suggested an appropriate magnetic field
\end{abstract}

Published in the Special Issue "Energy, Environment, Economics and Thermodynamics.

Wang Xiaodong

xiaodong.wang@ucas.ac.cn

1 College of Material Science and Opto-electronic Technology, University of Chinese Academic Sciences, Beijing 100049, China

2 CNRS-SIMAP-EPM PHELMA, BP75, 38402 Saint Martin d'Hères Cedex, France

3 UTC Bucharest, bd. Pache Protopopescu, Bucharest, Romania

4 State Key Laboratory of Advanced Steel Processing and Products, Central Iron and Steel Research Institute, Beijing 100081, China with optimized electromagnetic parameters for obtaining high-quality, low-defect casting products.

Keywords Heat and mass transfer - Solidification . Magnetic field · Lorentz force $\cdot$ Segregation

\section{Introduction}

Solidification is one of the main processes in the metallurgy industry in which heat and mass transfer play an essential role in the determination of casting metal/alloy quality, i.e., the micro and macro-structure, morphology, texture, mechanical properties, etc. [1-3]. The casting of pure metals is rare when compared with that of metallic alloys for both nonferrous and ferrous metals. Therefore, macrosegregation, also called solute segregation, cannot be avoided while solidifying due to solute rejection from the liquid phase.

During the processing of solute rich alloys, macrosegregation defects can result from the solidification step [4]. These defects are characterized by composition differences in the space domain on the scale of the product, which deteriorates the properties of the material. These defects were observed in various solidification processes, including forge ingots, vacuum arc remelted ingots, and directionally solidified turbine blades. The improvement of such macrosegregation is a central problem in the solidification process. In this paper, we clarify (a) how the fluid flow in the mushy zone transports the solute and generates the segregations; and (b) whether it is possible to control the fluid flow by means of an electromagnetic stirring device to avoid segregations.

Segregations are essentially generated by fluid motion within the mushy zone. This motion may be generated by 
three different mechanisms: (a) the thermodynamic equilibrium in the mushy region and the liquid solute concentration that exhibit gradients directly linked to the temperature gradient; thus, these gradients are responsible for the natural convection of solutes; (b) the electromagnetic forces can directly act on the liquid phase to generate a fluid motion within mush zone; and (c) the fluid motion in the liquid zone may create pressure variations along the solidification front which forces liquid flow inside the mushy zone, as is typical in a porous medium [5].

Lorentz force, is considered as an efficient means to change the flow and consequently influence solute redistribution. However, steady electromagnetic stirring does not suppress macrosegregations but only modifies their location. Such stirring may even create and promote macrosegregation. In the review paper, we have shown that a slow modulation of electromagnetic forces may much prevent the formation of macro-segregation $[6,7]$.

\section{A solidifying benchmark}

\section{Experimental configuration}

To better understand the underlying scientific principles of heat and mass transfer during alloy solidification, a HeleShaw-like, quasi-two-dimensional solidification benchmark experiment with well-controlled thermal boundary conditions was proposed $[8,9]$. The experiment consisted of solidifying a rectangular ingot of pure tin and binary Sn$3 \mathrm{wt} \% \mathrm{~Pb}$ alloy using two lateral heat exchangers to extract the heat flux from two sides of the solidifying sample. The temperature difference between the two sides of the heat exchangers may vary from 0 to $40 \mathrm{~K}$, and the cooling rate from 0.02 to $0.04 \mathrm{~K} / \mathrm{s}$. Segregation formation can be improved within above ranges. An array of fifty thermocouples was sit on the largest area walls to measure the instantaneous temperature distribution, which allowed us to visibly observe temperature field evolution during the whole solidification process, the heat and mass transfer process consequently was achieved, including phase transformations [8]. Meanwhile, a traveling magnetic field was exerted by a linear motor that was placed underneath the rectangular ingot [9]. This traveling magnetic field drives the melt by imposing the volumic and mechanically contactless Lorentz force on it. The role of imposing electromagnetic force and generating forced convection were also investigated in this solidifying benchmark.

\section{Experimental results}

Figure 1 shows the results of this experimental benchmark, which imply that heat and mass transfer played a dominating role during the solidification process. Clearly, this quasi-two-dimensional experimental benchmark demonstrates the evolution of heat and mass transfer during the binary $\mathrm{Sn}-3 \mathrm{wt} \% \mathrm{~Pb}$ alloy solidification process. Figure 1a shows the measurement results of the temperature field, which imply the influence of natural convection. The circle shows the natural convection trend as well as the dense isothermal contours on the left side, showing the beginning stage of the solidification process; Fig. 1b denotes the morphology of the grain contour in which the Columnar-to-Equiaxed Transition (CET) occurred in certain cooling rates and local temperature gradients; Fig. 1c represents an X-ray post-mortem result of the solidified ingot showing lead segregation (in white), and freckles are visible at the right lower corner of the sample, which naturally formed during the solidification; Fig. 1d demonstrates the Lorentz force having a significant effect upon the solidifying structure: it can be observed that the electromagnetic force has the capability to change the microstructure and, consequently, the CET process.

\section{Numerical investigation}

\section{A multiscale and multiphase numerical model}

A numerical model has been developed to accomplish two objectives: first, to better understand the underlying physical properties of the solidification mechanism, taking into account micro-scale crystal growth, meso-scale segregation, and the macro-scale momentum transport effects in a coupling manner; this study has been elaborated by Ciobanas and Fautrelle via numerical modeling, a multiphase Eulerian model for columnar and equiaxed dendritic solidification has been developed by those authors [10, 11]; and second, to investigate the ability of the periodically reversed Lorentz force on the improvement of solutal segregations at the interface of the solid/liquid interface [5].

\section{Effect of periodically reversed Lorentz force}

We investigated a two-dimensional rectangular solidifying domain with a pair of traveling magnetic fields imposing near the vertical walls of the solidifying ingot. The melt was chosen as a binary alloy $\mathrm{Pb}-10 \mathrm{wt} \% \mathrm{Sn}$. The detailed boundary conditions can be found in [5]. The numerical results of the concentration, indicating the solutal segregations, are illustrated in Fig. 2.

Note that the forced convection induced by normal electromagnetic stirring does not suppress the channel segregates but controls their location according to the liquid flow patterns in this example. Figure 2 demonstrates 


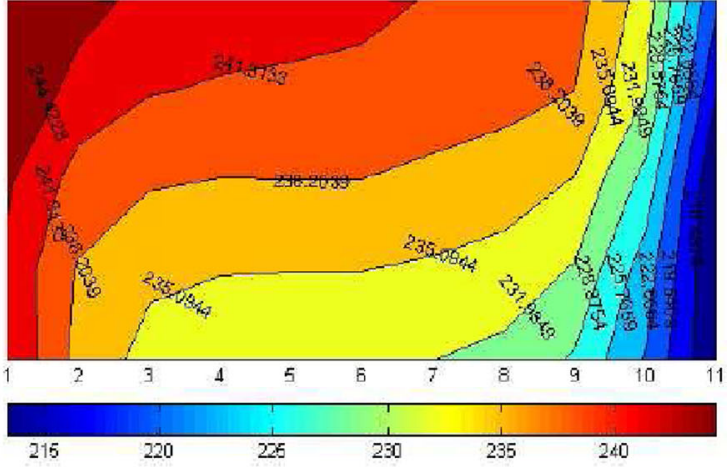

(a)

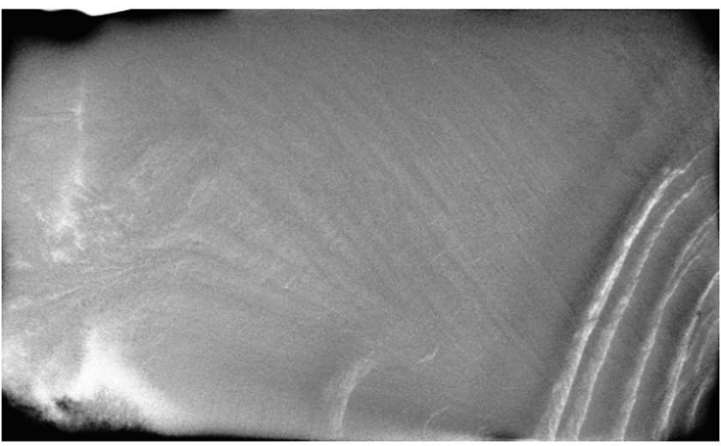

(c)

Fig. 1 A quasi-two-dimensional experimental benchmark showing the evolution of heat and mass transfer during a binary Sn-3 wt $\% \mathrm{~Pb}$ alloy solidification process. a Measurement results of the temperature field imply the action of natural convection; the dense isothermal contours at the lower right corner showing the beginning stage of solidification; b morphology of the grain contour; c post-mortem $\mathrm{X}$-ray of the solidified ingot showing lead segregation (in white);

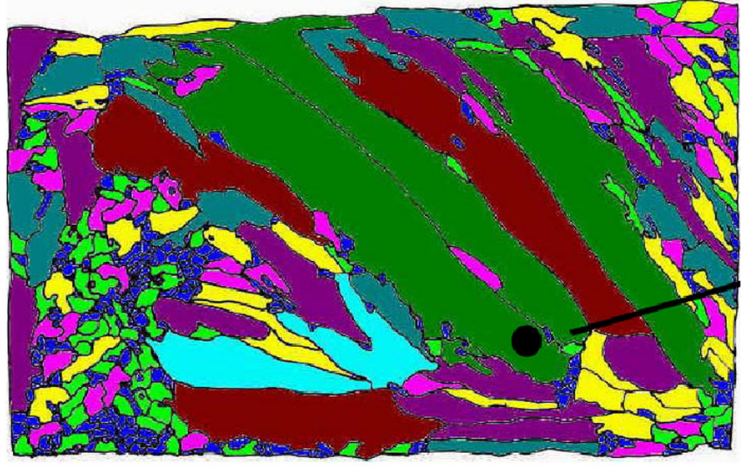

(b)

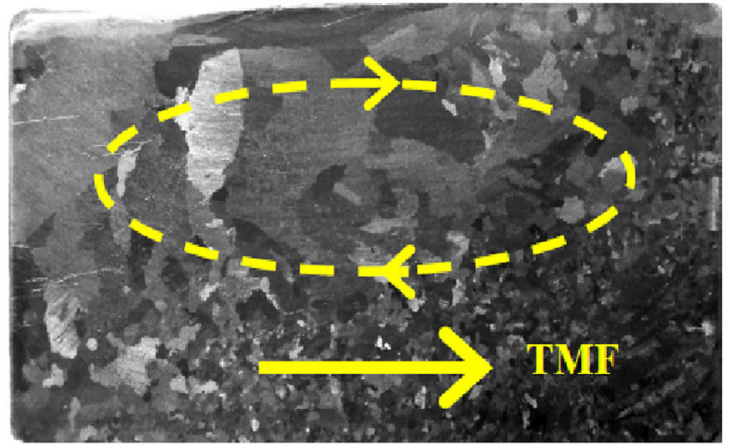

(d)

freckles are visible in the right part of the sample; $\mathbf{d}$ Lorentz force acting upon the solidification process. The rectangular ingot is $10 \mathrm{~cm}$ in length, $6 \mathrm{~cm}$ in height and $1 \mathrm{~cm}$ in width. The parameters of the traveling magnetic field are of the frequency $50 \mathrm{~Hz}$, the pole pitch $85 \mathrm{~mm}$, the wave number is $0.074 \mathrm{~mm}^{-1}$, and $B_{0}$ is approximately 30 $\mathrm{mT}[8,9]$

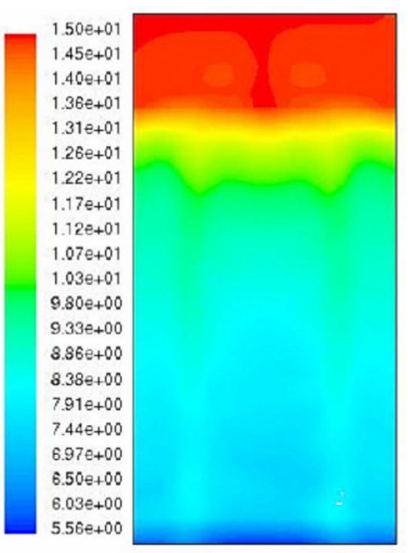

(c)

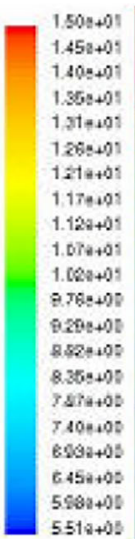

(b)

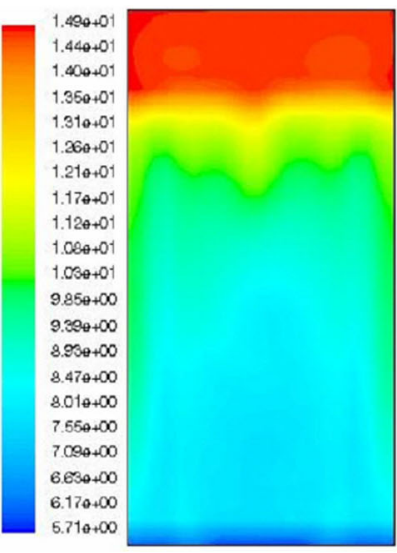

(a)

(b)

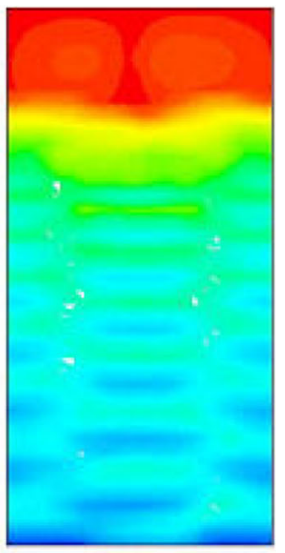

(d)

Fig. 2 Solidification of $\mathrm{Pb}-10 \mathrm{wt} \% \mathrm{Sn}$. Maps of the solute mean concentration for various modulation periods at $t=1200 \mathrm{~s}$. The cooling rate is $1 \mathrm{~K} / \mathrm{min}$. F0 $=103 \mathrm{~N} / \mathrm{m} 3$. a $p=4 \mathrm{~s}, \mathbf{b} p=12 \mathrm{~s}, \mathbf{c} p=32 \mathrm{~s}, \mathbf{d} p=128 \mathrm{~s}$ [5]

the improving effect of modulated traveling electromagnetic stirring on macrosegregation. It is shown that modulated electromagnetic stirring may suppress the segregated channels if the modulation period, Tm, is chosen properly. If the value of $\mathrm{Tm}$ is too short, the flow is not sensitive to Lorentz forces, and the segregation pattern is 
similar to that obtained in the natural convection case. The concentration maps illustrated in Fig. 2a show such a behavior. For a long modulation period, channels begin to form alternatively in the center and near the lateral walls, as in the steady stirring case illustrated in Fig. 2b, c. The corresponding segregation pattern keeps the memory of the channel formations and disappearances, and the lateral segregations are almost suppressed. The latter result may be interpreted by considering both the required flow establishment time and the transit time of the flow inside the mushy zone. Note that the normal segregation is also slightly reduced by the modulation when the optimal period is used. If the value of Tm is too long, macrosegregation is recreated, as shown in Fig. 2 d.

\section{A periodically reversed flow, driven by a modulated traveling magnetic field}

\section{Modulated magnetic field and MHD flow}

Based on the solidifying benchmark and the multiscale solidifying numerical modeling, it is necessary to experimentally investigate the flow behaviors driven by the socalled modulated magnetic field [6] or pulsed magnetic field $[12,13]$. An eutectic alloy GaInSn (melting temperature approximately equal to $10.5^{\circ} \mathrm{C}$ ), is used as the experimental metal and is contained in a transparent rectangular cavity with a width of $1 \mathrm{~cm}$, length of $10 \mathrm{~cm}$, and height of $6 \mathrm{~cm}$, respectively. A traveling magnetic field exerting by a linear induction motor, whose top surface is located $5 \mathrm{~mm}$ underneath the bottom of the cavity. Note that the classic way to reverse the direction of any traveling or rotating magnetic field motion is to permute two of the three phases. The frequency of the permutation is called the modulation frequency and is denoted by $f_{\mathrm{m}}$.

\section{Periodically reversed flow behaviors}

As shown in Fig. 3, a remarkably spatiotemporal organization of the velocity field develops in the presence of modulation. As $f_{\mathrm{m}}$ decreases $\left(f_{\mathrm{m}} \leq 2 \mathrm{~Hz}\right.$, Fig. 3a through $3 \mathrm{~d}$ ), the randomness disappears and periodic oscillations develop, whose frequencies converge to the modulation frequency. This organization can be dramatically altered by the unsteadiness of the driving force, but this requires that the typical modulation period is of the same order as, or larger than the transit time of the fluid particles in the main recirculation loop. When the modulation frequency is too large, typically $f_{\mathrm{m}} \gg 2 \mathrm{~Hz}$, the time available between two reversals is too short to allow for a change in the velocity direction and re-establishment of a similar flow organization with the opposite sign [6], therefore, there exists an optimizing modulating frequency for a certain solidifying configuration.

From the above analysis, with the help of a traveling magnetic field, a periodically reversed flow can be obtained by optimizing the modulation frequency for a certain rectangular ingot. However, from a practical point of view, the cylindrical geometry of the ingot is very popular, clearly, and more complex three-dimensional periodical flow behaviors need to be clarified at least in the azimuthal and the axial directions, which will be introduced in the next section.

\section{Flow patterns under the condition of a helical permanent magnetic field}

\section{A permanent helical magnetic field}

To achieve the use of a periodically reversed electromagnetic force to act upon the melt, which aims to improve solidifying defects, such as macrosegregation, in the more common casting ingot (cylindrical rather than rectangular), a helical permanent magnetic field has been designed and fabricated.

Considering the difficulty of directly constructing a whole, helical, permanent magnet, as well as the magnetizing process (see Fig. 4a), a method was adapted that incorporates a series of small, arc-shaped, permanent magnets, which were magnetized in their radii directions and were piled along the Archimedean space curve to form a special static magnetic field (see Fig. 4b). The bulk cylindrical liquid metal GaInSn is located inside of the electromagnetic stirrer, which was driven by a rotating motor. The modulated magnetic field can be attained by an alternating change in the rotating direction of the driving motor. Ultrasonic Doppler Velocimetry (UDV) was used to quantitatively measure the liquid metal flow driven by the electromagnetic stirrer. The size of the cylindrical pool is the follows: the diameter $2 R=40 \mathrm{~mm}$ and the height $H=70 \mathrm{~mm}$, additional details can be referred to in [14].

A helical magnetic field can be considered as a spatial superposition of rotating and traveling magnetic fields; the electromagnetic parameters of the traveling components are $B_{\mathrm{z}}=10 \mathrm{mT}$, the pole pitch in the $\mathrm{z}$ direction $P_{\mathrm{z}}=84 \mathrm{~mm}$, and the rotating components are $B_{\phi}=85 \mathrm{mT}$, the azimuthal wave number $\kappa_{\phi}=0.025 \mathrm{~mm}^{-1}$. Therefore, for such configuration, spatial flow can be expected. We measured the azimuthal and axial components of the velocity fields using UDV. To demonstrate three-dimensional flow behaviors of the liquid metal, we performed vector synthesis and velocity measurements at two moments $\left(t_{1}=21.2 \mathrm{~s}, t_{2}=23.6 \mathrm{~s}\right)$, which can be observed in Fig. 5. The experimental conditions are as follows: the rotating speed of the electromagnetic 


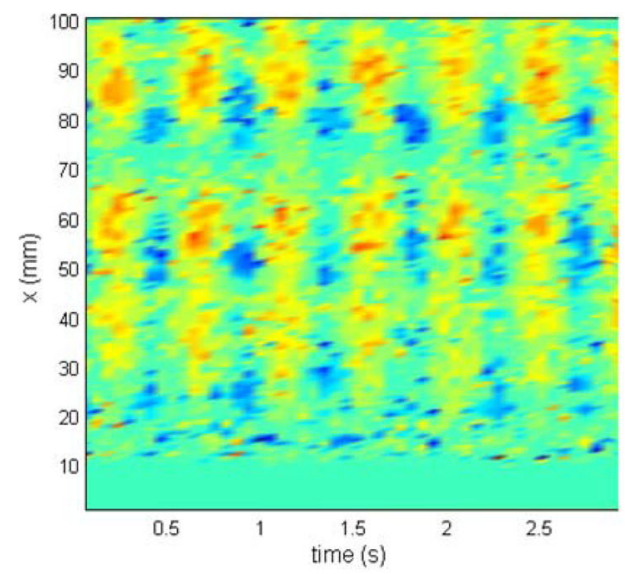

(a) $f_{\mathrm{m}}=2 \mathrm{~Hz}$

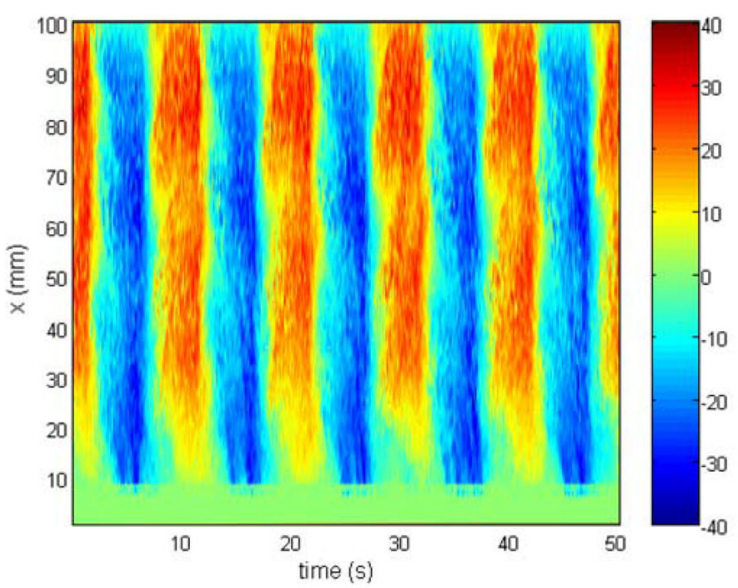

(c) $f_{\mathrm{m}}=0.1 \mathrm{~Hz}$

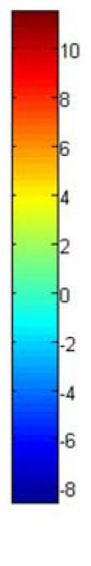

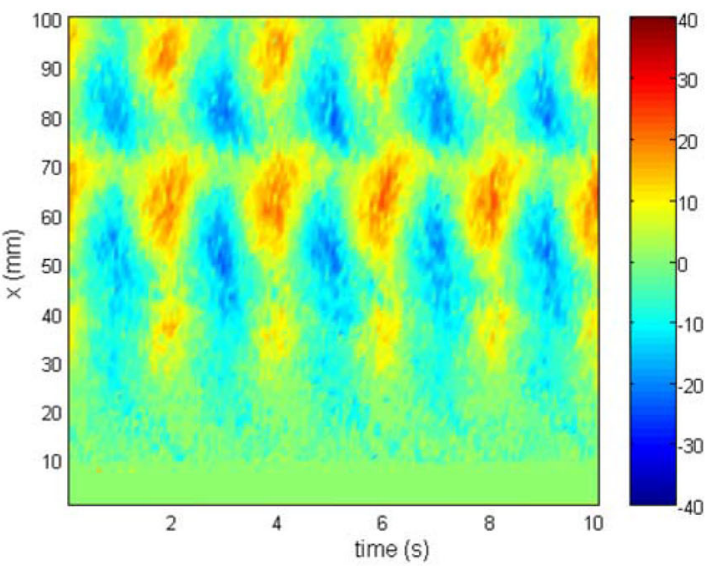

(b) $f_{\mathrm{m}}=0.5 \mathrm{~Hz}$

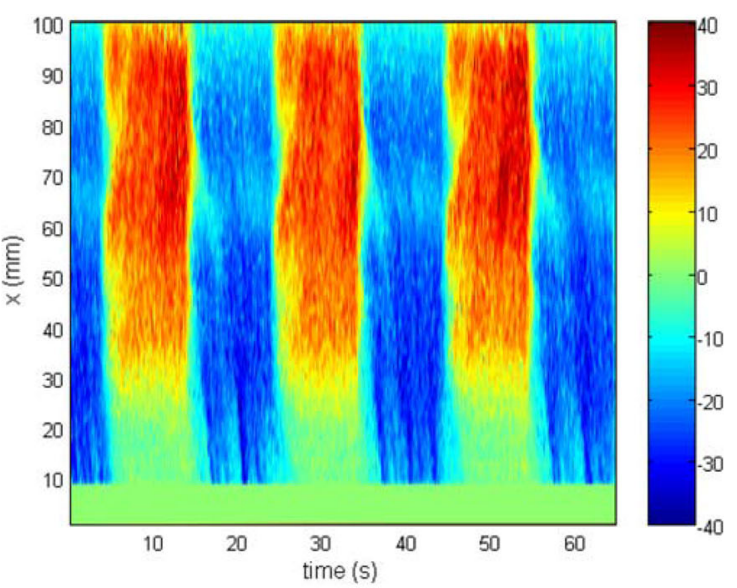

(d) $f_{\mathrm{m}}=0.05 \mathrm{~Hz}$

Fig. 3 Spatiotemporal evolution of the velocity fluctuation profiles in the $\mathrm{x}$ direction for various modulation frequencies: a $f_{\mathrm{m}}=2 \mathrm{~Hz}$, b $f_{\mathrm{m}}=0.5 \mathrm{~Hz}, \mathbf{c} f_{\mathrm{m}}=0.1 \mathrm{~Hz}$, and $\mathbf{d} f_{\mathrm{m}}=0.05 \mathrm{~Hz}$ [6]. The pseudo-color maps represent the amplitude of the velocity in $\mathrm{mm} / \mathrm{s}$

Fig. 4 Comparison between the structure of an ideal helical permanent magnet (a) and the actual helical magnet constructed with permanent magnet units (b)

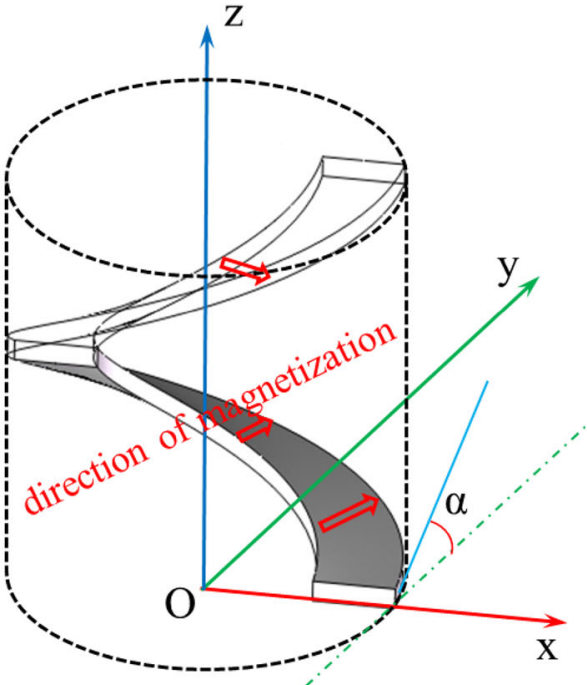

(a)

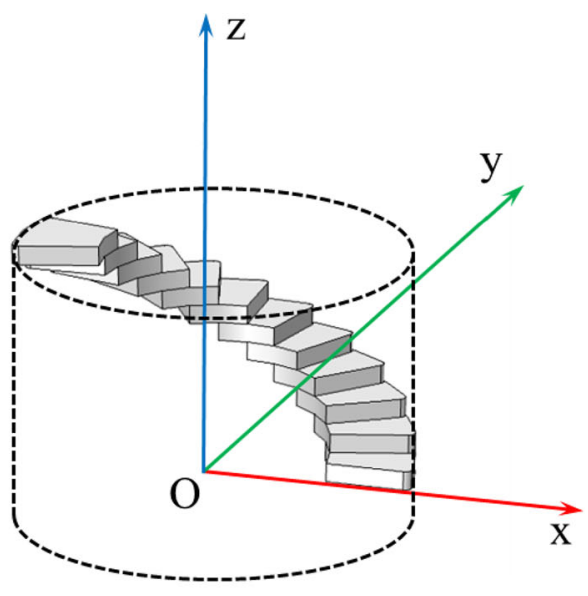

(b) 
Fig. 5 Three-dimensional liquid metal flow driven by a modulated helical magnetic field at $t_{1}=21.2 \mathrm{~s}(\mathbf{a})$ and $t_{2}=23.6 \mathrm{~s}(\mathbf{b})$ with $\omega=8 \pi$ $\mathrm{rad} / \mathrm{s}$ and $f_{\mathrm{m}}=0.2 \mathrm{~Hz}$ [14]

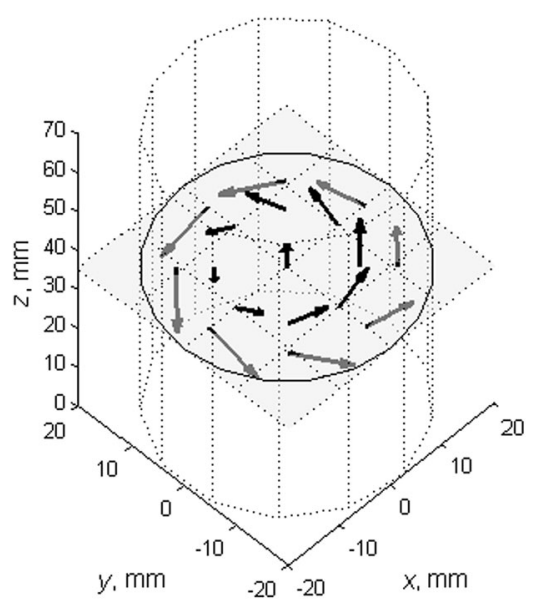

(a)

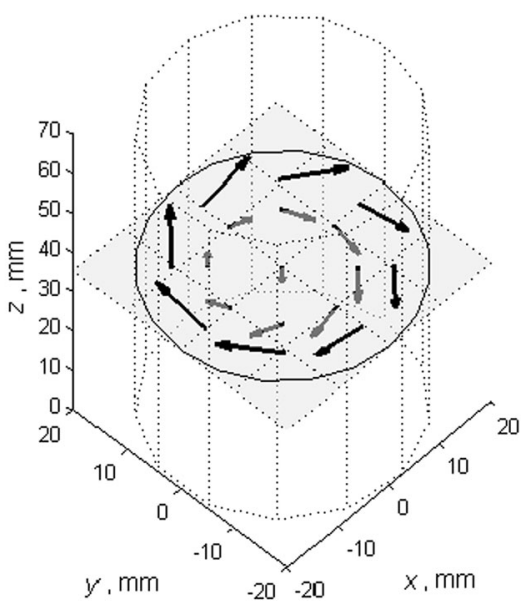

(b) stirrer is $\omega=8 \pi \mathrm{rad} / \mathrm{s}$, the modulation frequency $f_{\mathrm{m}}=0.2 \mathrm{~Hz}$, and the velocity vectors were shown at the plane of $z=35 \mathrm{~mm}$. Note that the measurement time $\left(t_{1}=21.2 \mathrm{~s}\right)$ in this experiment was in the first half period of the modulated magnetic field, and $\left(t_{2}=23.6 \mathrm{~s}\right)$ in the second half. The gray arrows in Fig. 5 indicate that it is located under the plane of $z=35 \mathrm{~mm}$, and the black arrows are above the plane of $z=35 \mathrm{~mm}$. It is easily observed that the liquid metal flow spins up in Fig. 5a and that the flow spins down in Fig. 5b. These measurement results indicate that a periodical swirling flow has been driven and developed by the helical permanent magnetic field.

\section{Effect of improving macrosegregation}

To justify the macrosegregation-improving effect by imposing the modulated magnetic field, we present an additional result under the conditions described in "Effect of periodically reversed Lorentz force" [5]. Figure 6 shows the horizontal profiles of the mean solute concentration averaged along a vertical line for various electromagnetic force modulation periods. With the optimal modulation period ( $\sim 18 \mathrm{~s})$, the macrosegregation was observed to be almost suppressed both in the vicinity of the wall and at the center portion. This result reveals that the electromagnetic field, if designed meticulously, can significantly improve the solidification defects, such as macrosegregation.

\section{Conclusion}

An experimental benchmark has proven its ability to obtain well-controlled solidification and demonstrated that heat and mass transfer in the liquid and mushy zone determines the solidification quality and dominates the solutal segregation via diffusion and convection effects. The numerical simulation of the directional solidification of a binary $\mathrm{Pb}$ Sn alloy [5] actually demonstrated that macrosegregation could be suppressed by a vortex flow when the direction is periodically reversed. This analysis aims to provide guidelines on how to design an inductor to drive the most efficient flow in a realistic experiment. According to our strategy, a few final remarks can be made. It is clear that
Fig. 6 Horizontal profiles of the mean solute concentration averaged along a vertical line for various modulation periods of the electromagnetic forces. Case of $\mathrm{Pb}-\mathrm{Sn} 10 \%$ wt. $F_{0}=10^{3} \mathrm{~N} / \mathrm{m}^{3}[5]$

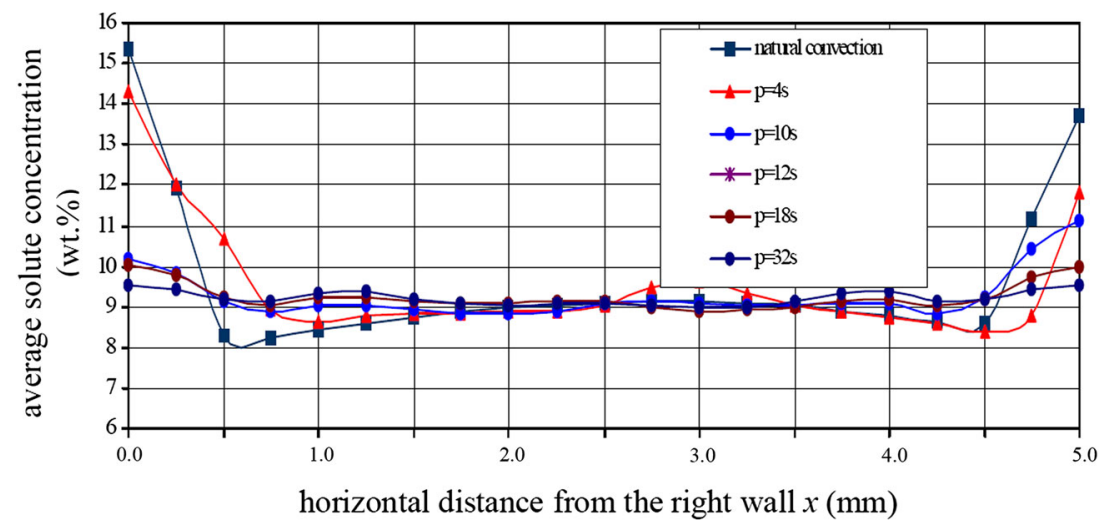


controllable electromagnetically driven flow can be obtained. The quantitative results are established for a parallelepipedic enclosure and further for a common cylindrical container. There may still be some interest in practical geometries, for example, in cylindrical directional solidification furnaces.

Acknowledgments XDW acknowledges financial support from the Program of "One Hundred Talented People of Chinese Academy of Sciences" (Y32901FEA2), NSFC (51374190), and CAS-Space science (XDA04078400).

Open Access This article is distributed under the terms of the Creative Commons Attribution 4.0 International License (http://creativecommons.org/licenses/by/4.0/), which permits unrestricted use, distribution, and reproduction in any medium, provided you give appropriate credit to the original author(s) and the source, provide a link to the Creative Commons license, and indicate if changes were made.

\section{References}

1. Flemings, M.C., Nereo, G.E.: Macrosegregation: part 1. Trans. Met. Soc. AIME 239, 1449 (1967)

2. Hultgren, A.: A and V segregation in killed steel ingots. Scand. J. Metall. 2, 217 (1973)

3. Engström, G., Fredriksson, H.: On the mechanism of macrosegregation formation in continuously cast steels. Scand. J. Metall. 12, 3 (1983)

4. Sarazin, J.R., Hellawell, A.: Channel formation in $\mathrm{Pb}-\mathrm{Sn}, \mathrm{Pb}-\mathrm{Sb}$, and $\mathrm{Pb}-\mathrm{Sn}-\mathrm{Sb}$ alloy ingots and comparison with the system $\mathrm{NH}_{4} \mathrm{Cl}-\mathrm{H}_{2} \mathrm{O}$. Metall. Trans. 19A, 1861-1871 (1988)

5. Wang, X.D., Ciobanas, A., Baltaretu, F., Bianchi, A.M., Fautrelle, Y.: Control of the macrosegregation during solidification of a binary alloy by means of a AC magnetic field. Mater. Sci. Forum 508, 163-168 (2006)

6. Wang, X.D., Fautrelle, Y., Etay, J., Moreau, R.: A periodically reversed flow driven by a modulated traveling magnetic field. Part I: Experiments with GaInSn. Metall. Mater. Trans. B 40B, 82-90 (2009)

7. Wang, X.D., Moreau, R., Etay, J., Fautrelle, Y.: A periodically reversed flow driven by a modulated traveling magnetic field. Part II: theoretical model.. Metall. Mater. Trans. B 40B, 104-113 (2009)

8. Wang, X.D., Fautrelle, Y.: An investigation of the influence on tin solidification using a quasi- two- dimensional experimental benchmark. Int. J. Heat Mass Transfer. 52, 5624-5633 (2009)

9. Hachani, L., Saadi, B., Wang, X.D., Fautrelle, Y., et al.: Experimental analysis of the solidification of $\mathrm{Sn}-3 \mathrm{wt} \% \mathrm{~Pb}$ alloy under natural convection. Int. J. Heat Mass Transfer. 55, 1986-1996 (2012)

10. Ciobanas, A.I., Fautrelle, Y.: Ensemble averaged multiphase Eulerian model for columnar/equiaxed solidification of a binary alloy: I. The mathematical model. J. Phys. D Appl. Phys. 40, 3733 (2007)

11. Ciobanas, A.I., Fautrelle, Y.: Ensemble averaged multi-phase Eulerian model for columnar/equiaxed solidification of a binary alloy: II. Simulation of the columnar-to-equiaxed transition (CET). J. Phys. D Appl. Phys. 40, 4310 (2007)

12. Eckert, S., Nikrityuk, P.A., Raebiger, D., Eckert, K., Gerbeth, G.: Efficient melt stirring using pulse sequences of a rotating magnetic field: Part I. Flow field in a liquid metal column. Metall. Mater. Trans. B 38B, 977-988 (2007)

13. Willers, B., Eckert, S., Nikrityuk, P.A., Raebiger, D., Dong, J., Eckert, K., Gerbeth, G.: Efficient melt stirring using intermittent or alternating rotating magnetic fields: II-Application during Solidification of Al-Si Alloys. Metall. Mater. Trans. B 39B, 304-316 (2008)

14. Wang, S.W., Wang, X.D., Ni, M.J., Zhang, X.D., Wang, Z.H., $\mathrm{Na}$, X.Z.: Liquid metal flow driven by a modulated helical magnetic field. Acta Metallurgica Sinica (in Chinese). 49(5), 544-552 (2013) 